Article

\title{
A Fundamental Consideration of Active Noise Control System by Small Actuator for Ultra-Compact EV
}

\author{
Taro Kato ${ }^{1}$, Ryosuke Suzuki ${ }^{1}$, Rina Miyao ${ }^{1}$, Hideaki Kato ${ }^{2, *}$ and Takayoshi Narita ${ }^{2}$ (D) \\ 1 Course of Mechanical Engineering, Tokai University, Kitakaname 4-4-1, Hiratsuka-shi, \\ Kanagawa 259-1292, Japan; kato.taro19910726@gmail.com (T.K.); 8bemm048@mail.u-tokai.ac.jp (R.S.); \\ 8bemm079@mail.u-tokai.ac.jp (R.M.) \\ 2 Department of Prime Mover Engineering, Tokai University, Kitakaname 4-4-1, Hiratsuka-shi, \\ Kanagawa 259-1292, Japan; narita@tsc.u-tokai.ac.jp \\ * Correspondence: hkato@tokai-u.jp; Tel.: +81-463-58-1211
}

Received: 15 June 2018; Accepted: 13 August 2018; Published: 16 August 2018

\begin{abstract}
The ultra-compact electric vehicle has recently experienced increasing popularity for short-distance travel. However, one of the issues with ultra-compact electric vehicles is that although the engine is silent, exterior road and wind noise have a significant impact on the occupant's comfort in the interior space. We propose an ANC system whereby a kind of small actuator is installed on the roof of an ultra-compact electric vehicle. In this paper, we consider the noise control effects of using a giant magnetostrictive actuator and conduct an experimental study on feed-forward and feedback control systems.
\end{abstract}

Keywords: ultra-compact electric vehicle; active noise control; giant magnetostrictive actuator; all-pass filter; adaptive filter

\section{Introduction}

In recent years, one- or two-seater ultra-compact electric vehicles, which have become popular for short-distance travel, have been researched and actively developed [1]. Ultra-compact electric vehicles can easily make turns owing to their compactness and are also environment-friendly. On the other hand, while the engines of ultra-compact electric vehicles are silent, exterior road and wind noises have a large impact on occupant comfort in the interior space. Road noise, in particular, is a low-frequency noise and represents a challenge for conventional passive noise reduction technologies that use sound-absorbing materials or reflecting plates, which increase weight and reduce available interior space. Recently, interior noise reduction by active noise control (ANC) has been widely used in the automobile industry [2]. Generally, an installed ANC system for the vehicle uses a dedicated speaker or mechanical equipment to generate and control a sound wave. The ultra-compact electric vehicle that we intend to use is required to use a small and light device that ensures the interior space of the lightweight vehicle more strongly than a conventional vehicle.

On the other hand, an ANC system that uses a small and light device instead of a speaker is conducted [3,4]. Features of these ANC systems include the use of several piezoelectric type actuators and installed corrective locations for objects. However, when we consider installing an ANC system that uses piezoelectric type actuators in an ultra-compact electric vehicle, we cannot install this system because we face several issues regarding secure visibility. Nonetheless, we installed several actuators on the front or side window and generated enough power for one actuator. Therefore, we focused on a giant magnetostrictive actuator among the small and light devices. The feature of this giant 
magnetostrictive actuator is better than the piezoelectric type actuator regarding the generation of power for one actuator and greater durability.

Our group aimed to create an ANC system that targets the location of the ears of a passenger for low frequency noise, such as load noise from outside generated by the surface vibration from using a giant magnetostrictive actuator. However, there has been little research on an ANC system that uses giant magnetostrictive actuators. Furthermore, since the sound environment of the cabin changes in the actual condition, it is important to consider the possibility of applying the adaptive filter with an ANC system using the giant magnetostrictive actuator in response to that change. In this paper, as the initial research stage of the ANC system, we installed an apparatus on the rooftop of an ultra-compact electric vehicle to actively cut the interface vibration for a single frequency, assuming low frequency noises such as road noise that are transmitted inside the vehicle. We attempted a basic study on the creation of an ANC system and performed a feed-forward control system that used an all-pass filter to develop a feedback control system using an adaptive filter that can automatically compensate between noise and control waves, for the purpose of investigating interior noise reduction.

\section{Noise Reduction Experiment by Using A Giant Magnetostrictive Actuator}

\subsection{Experimental Equipment and Noise Reduction Method}

In this experiment on noise reduction, we used the exterior roof of an ultra-compact vehicle (2040 mm length, $1200 \mathrm{~mm}$ width, $1590 \mathrm{~mm}$ height), shown in Figure 1, to study noise reduction using a giant magnetostrictive actuator. The single frequency noise coming from outside the vehicle, which is assumed as road noise, was produced by a speaker installed underneath the vehicle to simulate the condition of actual road noise originating from the outside. To create the ideal condition inside the vehicle, which excludes the effect of a secondary sound field in the vehicle, we attached an acoustic sponge approximately $30 \mathrm{~mm}$ thick on the inner surface, except on the windows and floor, so that we could exclude noise reflected from inside the roof of the vehicle. Figure 2 shows a schematic of the noise reduction experiment. A s-BOX manufactured by MTT was used as the digital signal processor (DSP). An AT9944 microphone manufactured by Audio-technica, designated as Sensor 1, was used to measure the noise generated during the experiment. In addition, we used an ECM-CR120 manufactured by Sony, designed as Sensor 2, to measure the noise reduction inside the vehicle. A giant magnetostrictive actuator was installed vertically on the rooftop to generate the control sound wave as shown in Figure 3. We show the specification detail of using a giant magnetostrictive actuator as Table 1. In this paper, we selected a single frequency sound wave of $200 \mathrm{~Hz}$ and $300 \mathrm{~Hz}$ in the road noise frequency range between $100 \mathrm{~Hz}$ and $500 \mathrm{~Hz}$ as a basic consideration of the ANC system by using a giant magnetostrictive actuator [5-8]. We measured the single frequency noise, which is from a speaker under the vehicle with Sensor 1, installed at the front lower part of the vehicle. The measured noise generates a control signal through the DSP. Then, a control sound wave was generated by the excitation of the roof with the giant magnetostrictive actuator that controlled the noise. It is a calculation amount that can be operated at $1 \mathrm{kHz}$ with the DSP used this time. The noise reduction volume was evaluated with Sensor 2, installed at $x=600 \mathrm{~mm}$ and $y=14500 \mathrm{~mm}$ near the center of the vehicle ( $30 \mathrm{~mm}$ from gateway), which was assumed as the location of the passenger's ears and complies with the JIS standard. This study focused on muffling around the ears of passengers, therefore, the sound pressure except around the ears was not evaluated. 




Figure 1. Ultra-compact electric vehicle.

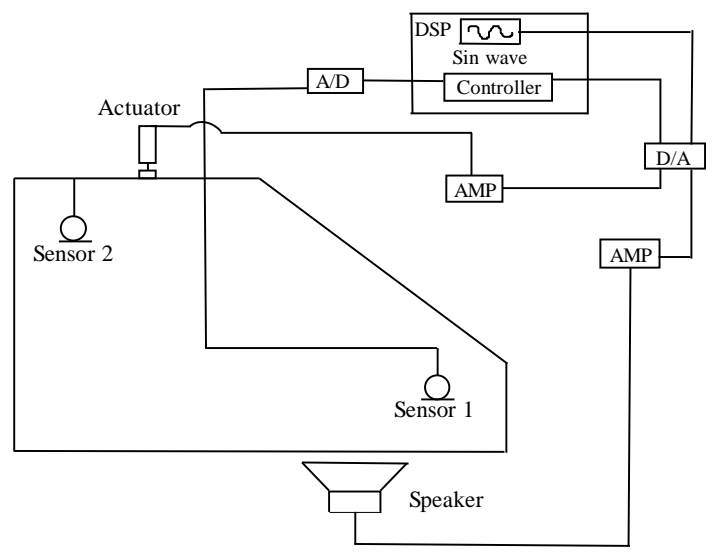

Figure 2. Control system.

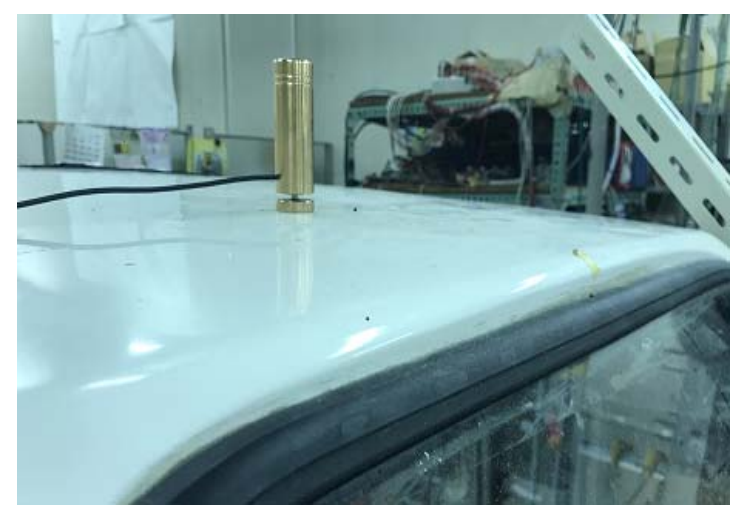

Figure 3. Installation of giant magnetostrictive actuator.

Table 1. Specification of giant magnetostrictive actuator.

\begin{tabular}{cc}
\hline Giant Magnetostrictive Actuator & Value \\
\hline Size $(\mathrm{mm})$ & 90 \\
Weight $(\mathrm{g})$ & 390 \\
Magnetostriction $\lambda(\mathrm{ppm})$ & $\sim 2000$ \\
Generated stress $\sigma(\mathrm{N})$ & $\sim 30,000$ \\
Response speed $\mu(\mathrm{s})$ & $\sim 40$ \\
Electrical-mechanical coupling coefficient $K$ & $0.7 \sim 0.75$ \\
Energy density $\left(\mathrm{kJ} / \mathrm{m}^{3}\right)$ & $14 \sim 25$ \\
Curie temperature $\left({ }^{\circ} \mathrm{C}\right)$ & 350 \\
Electrical resistance $R(\Omega \mathrm{m})$ & $60 \times 10^{-8}$ \\
Density $\rho\left(\mathrm{kg} / \mathrm{m}^{3}\right)$ & $9.25 \times 10^{3}$ \\
Young's modulus $\mathrm{YH}(\mathrm{GPa})$ & 26.5 \\
Compressive strength $T_{c}(\mathrm{MPa})$ & 700 \\
\hline
\end{tabular}




\subsection{Noise Reduction Experiment by Using All-Pass Filter}

Figure 4 shows a block diagram of the feed-forward noise control system using an all-pass filter. A reference signal $a$ of the noise output from the speaker was detected by Sensor 1. A control signal $b$ for the phase compensator was generated by an all-pass filter $G(s)$, and amplified by gain $K$ [9]. A control sound wave was then generated by the giant magnetostrictive actuator installed on the roof so that the control signal could be reduced with Sensor 2. $P$ represents the path from the roof to Sensor 2 [10]. In the figure, $d$ is the sound pressure signal by which noise passes from Sensor 1 through thecommunication channel $P$ to Sensor 2 . In the feed-forward control, an error signal $e 1$, which is the difference between the noise and the control sound wave, is minimized in Sensor 2 . The gain $K$ was set to 80 , which was determined by trial and error in order to identify the position at which the noise reduction effect was maximized by the actuator.

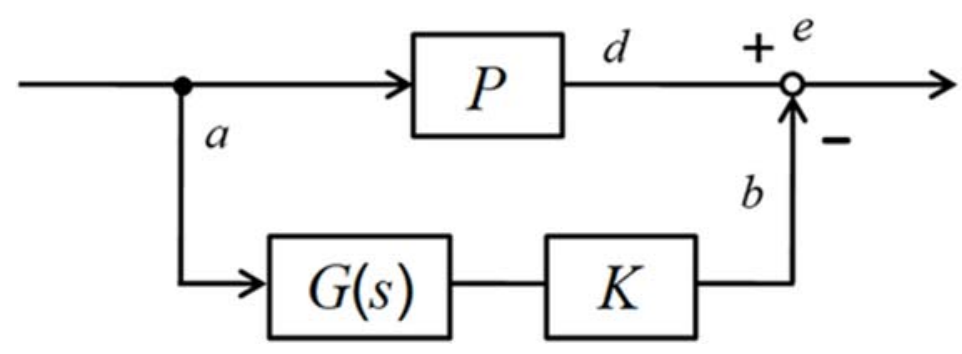

Figure 4. Block diagram of the All-pass filter.

\subsection{Experimental Result}

In these figures, the red line and blue line show the results without and with the control, respectively. The noise sound pressure level was set to approximately $80 \mathrm{~dB}$ to suppose the worst road noise environment that could introduced from outside the vehicle that reaches Sensor 2 in the vehicle. The noise coming from the outside was set to $200 \mathrm{~Hz}$ and $300 \mathrm{~Hz}$. From the experimental result shown in Figure 5, a noise reduction to $9.1 \mathrm{~dB}$ was achieved with the control, compared with $82.0 \mathrm{~dB}$ without the control, for an introduced noise frequency of $200 \mathrm{~Hz}$. In Figure 6, a noise reduction to $4.6 \mathrm{~dB}$ was achieved with the control, compared with $78.8 \mathrm{~dB}$ without control, for an introduced noise frequency of $300 \mathrm{~Hz}$. The comparison of the experimental results in Figure 7 shows that the noise was interrupted by the all-pass filter at $200 \mathrm{~Hz}$ and $300 \mathrm{~Hz}$.



Figure 5. All-pass filter $200 \mathrm{~Hz}$. 


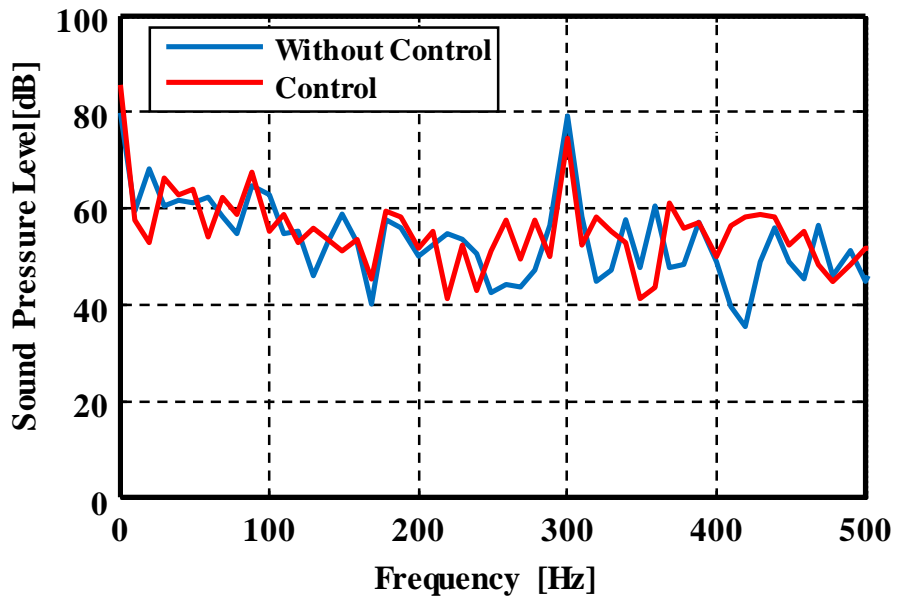

Figure 6. All-pass filter $300 \mathrm{~Hz}$.

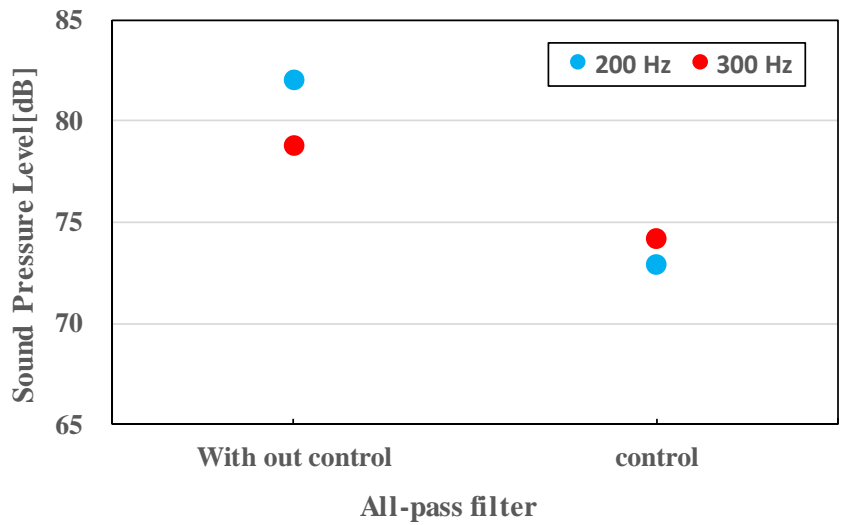

Figure 7. Experimental result of All-pass filter.

\section{Fundamental Consideration of Feedback ANC System}

In Section 2, we created an ANC system using a giant magnetostrictive actuator installed on the rooftop of a vehicle, which was understood to have a sufficient noise reduction effect for single-frequency noise. However, the feed-forward control system by the all-pass filter must be considered to determine the phase between the noise and control sound wave by trial and error. Therefore, we tried to create a new ANC system by using an adaptive filter.

\subsection{Noise Reduction Test by Using Adaptive-Pass Filter}

Figure 8 shows a block diagram of the feedback noise control system using an adaptive filter. A reference signal $a$ of the noise output from the speaker was detected by Sensor 1. The signal was detected through $S h$ and $C$, which are secondary pathways, that calculate the noise state from $S h$ and $e 2$ by the LMS algorithm, respectively. The control signal $b 2$, which changed the phase of $C$, created weighting coefficients by the LMS algorithm in the finite impulse response filter using the signal via second pass $C$. Then, control signal $b 2$ was output from a giant magnetostrictive actuator as a control sound wave. In the feedback control, an error signal $e 2$, which is the difference between the noise and control sound wave, was minimized in Sensor 2 to obtain the error signal. We used an AT9944 microphone manufactured by Audio-technica as Sensor 2. In this system, the feedback control was updated by the LMS algorithm to automatically minimize the error using the obtained $e 2$ in Sensor 2 [5-8]. The experimental procedure and setup were the same as the previous experiment in Section 2. 


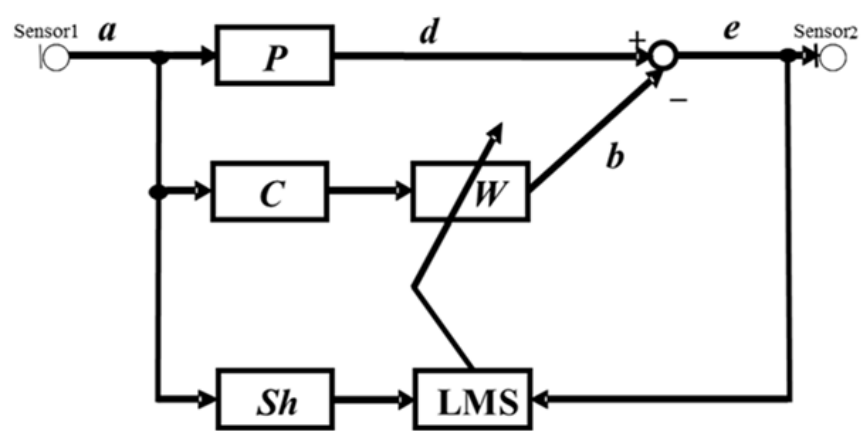

Figure 8. Block diagram of adaptive filter.

\subsection{Experimental Result}

The noise reduction results in Figures 9 and 10 show the power spectrum of the sound pressure, as obtained with Sensor 2. Figure 9 shows that a noise reduction to $11.4 \mathrm{~dB}$ was achieved with the control, compared with $81.7 \mathrm{~dB}$ without the control, for an introduced noise frequency of $200 \mathrm{~Hz}$. Figure 10 shows that a noise reduction to $7.3 \mathrm{~dB}$ was achieved with the control, compared with $83.6 \mathrm{~dB}$ without the control, for an introduced noise frequency of $300 \mathrm{~Hz}$. In these experiments, the convergences of the noise reduction effect have appeared within $0.3 \mathrm{~s}$ after the control system was started every time. These results indicate that the feedback control system by LMS algorithm is more effective in noise reduction than the feed-forward control system shown in Section 2 for only a single frequency. The comparison of experimental results in Figure 11 shows that the noise was interrupted by the adaptive filter at $200 \mathrm{~Hz}$ and $300 \mathrm{~Hz}$.



Figure 9. Adaptive filter $200 \mathrm{~Hz}$.



Figure 10. Adaptive filter $300 \mathrm{~Hz}$. 




Figure 11. Experimental result of adaptive filter.

\section{Conclusion}

In this paper, we proposed an ANC system using an all-pass filter and adaptive filter whereby a type of small actuator called a giant magnetostrictive actuator is installed on the rooftop of an ultra-compact electric vehicle. The experimental results showed that a feedback control system by the LMS algorithm is more effective in noise reduction than the feed-forward control system for only a single frequency. In this report, we conducted experiments to report that muffling experiments can be performed using a giant magnetostrictive actuator. For that reason, we believe that we must also conduct experiments on the reduction of assumed road noises in various frequency ranges and actual road noise in the future. Additionally, it is necessary to consider the various sound noise level and LMS convergences. However, in an actual vehicle, the condition of the model changes depending on the driving environment. It is conceivable that the frequency of the disturbance, the characteristics of the sound field, the sensor, or the actuator change during operation of the system. It is necessary to study the robustness of the proposed system in more detail against such changes in experimental conditions. Furthermore, from the viewpoint of improving the in-car environment of the ultra-small electric vehicle, we aim to construct an in-car environment that is tailored to each passenger, and to create an optimum in-car sound environment by quantitatively measuring the condition using a passenger's biometric information. We will continue research aiming at construction.

Author Contributions: T.K., R.S., R.M., T.N. and H.K. wrote the paper; T.K., R.S. and R.M. carried out experiments; T.K., R.S. and R.M. contributed the analyses of the data; T.N. and H.K. contributed the design of the controller and electric circuit.

Funding: This research received no external funding.

Conflicts of Interest: The authors declare no conflict of interest. The founding sponsors had no role in the design of the study; in the collection, analyses, or interpretation of data; in the writing of the manuscript, and in the decision to publish the results.

\section{References}

1. The Ministry of Land, Infrastructure, Transport and Trourism, The Guideline Towards Ultra-Compact Mobility. Available online: http:/ / www.mlit.go.jp/common/000212867.pdf (accessed on 16 August 2018).

2. Sunaga, D.; Aono, S.; Matsumoto, H.; Teramura, Y.; Kubota, H. A study on the effectiveness of micro electric vehicle in the metropolitan suburbs. Infrastruct. Plann. Manage. 2016, 72, 641-651. [CrossRef]

3. Sanada, A.; Tanaka, N. Active control of sound transmission through a honeycomb panel with feedforward and feedback control. Mech. Eng. J. 2016, 3, 16-00466. [CrossRef]

4. Meysam, S.M.; Chin, Y.T.; Xiang, Y.; Lei, Z.; Shuting, C.; Kui, Y.; Fangsen, C.; Sai, M.P.; Shyh, H.C.; Sze, T.T. Transparent piezoelectric film speakers for windows with active noise mitigation function. Appl. Acoust. 2018, 137, 90-97. 
5. Yokota, K.; Koba, Y.; Ishikawa, S.; Kijimoto, S. Active noise control for a moving evaluation point considering the change of acoustic transfer function. Trans. JSME 2018, 84, 17-00446. [CrossRef]

6. Kanazawa, L.; Yasui, K.; Yaginuma, K. The reduction effect of construction machinery noise by feedforward type active noise control. Jpn. Archit. Inst. Environ. Pap. 2017, 82, 97-103. [CrossRef]

7. Markus, E.C.; Juergen, H.Z. Active Noise Control by Adaptive Noise Filtering. U.S. Patent 15,380,319, 22 June 2017.

8. Nitin, K.; Jon, D.H.; John, L.M. Systems and Methods for Adaptive Active Noise Cancellation for Multiple-Driver Personal Audio Device. U.S. Patent 10,013,966, 3 July 2018.

9. Bisnette, J.B.; Smith, A.K.; Vipperman, J.S.; Budny, D.D. Active Noise Control Using Phase Compensated, Damped Resonant Filter. J. Vib. Acoust. 2005, 128, 148-155. [CrossRef]

10. Nishimura, M.; Usagawa, T.; Ise, S. Active Noise Control, 1st ed.; Corona Publishing Co., Ltd.: Tokyo, Japan, 2006; pp. 9-10.

(C) 2018 by the authors. Licensee MDPI, Basel, Switzerland. This article is an open access article distributed under the terms and conditions of the Creative Commons Attribution (CC BY) license (http:/ / creativecommons.org/licenses/by/4.0/). 